\title{
Monkey business in space
}

Sir - The News item on NASA's Bion programme of flying restrained and heavily instrumented rhesus macaques into space portrayed it in a light that the programme does not deserve (Nature 387, 4; 1997). In fact, Bion's design is such that meaningful results about "how musculoskeletal and regulatory mechanisms respond to and recover from space flight" (Bion 11/12 Integrated Science Proposal and Discipline Proposals, 1996) are virtually precluded.

Both restraint and microgravity result in osteopenia and disuse atrophy of muscles, which makes it impossible to determine the specific impact of microgravity (or any other cosmic factor) on bones and muscles in the restrained animals sent into space.

The 'regulatory physiology' protocol contains no hypotheses, which, in conjunction with the interference from restraint and psychological stress, the sample size of two, and dramatic individual differences in response (as documented by previous missions), reduces Bion to 'parametric tinkering', generating a spiral of inconclusive animal studies that are only vaguely, if at all, applicable to free-moving, relatively comfortable astronauts.

To be most useful for future missions, experimental data of this nature should be obtained from humans. The head-down tilt bed rest model simulates the physiological effects of microgravity and accurate noninvasive techniques, and osteoblast cultures are now available to study the cellular and biochemical mechanisms of osteopenia. All the musculoskeletal tests and eight out of nine 'regulatory physiology' tests could have been done in consenting humans. Eight measurements taken from astronauts are far more valuable than nine from straitjacketed monkeys in pain and distress.

We believe that sending animals into space is one of the most ineffective ways of studying the effects of microgravity on astronauts, and that subjecting conscious mammals to extreme trauma in the name of technological progress is unethical.

Bion involved some of the most invasive instrumentation ever imposed on a conscious primate. In addition to extensive wiring of the body and 10 incisions (including a perforation of abdominal muscles), each of the two flight and 10 control macaques had 11 holes (eight for Evarts crown, three for epidural cannulae) drilled in the skull roof and two depressions and canals in the zygomatic bones for an electro-oculogram. As well as the pain and terror of being strait-jacketed in these circumstances, the flight animals were exposed to motion sickness and shock from the dramatic concomitance of launch and landing. After returning to Earth, one macaque choked to death under anaesthesia. David O. Wiebers

Andrzej Elzanowski

Paul W. Gikas

Jennifer Leaning

Roger D. White

Scientific Advisory Council to

The Humane Society of the United States,

2100 L Street NW,

Washington, DC 20037, USA

e-mail:elzanow@ix.netcom.com
Some authors consider that a gene includes the regulatory sequences required for its expression ${ }^{1}$. But genes do not have to be expressed to be present. The somatic cells of a multicellular organism all have the same genes, but particular cell types express only some of them.

Inclusion of regulatory sequences expands the term 'gene' from a specification of 'what it is' to indicate also 'how it is used'. It may be useful to separate these two concepts. It is thought that major evolutionary changes in the morphology of organisms have resulted from altered patterns of synthesis of the same gene products ${ }^{2}$. It seems reasonable to consider that the genes themselves have not changed, but rather the ways in which they have been used.

Inclusion of regulatory sequences introduces considerable complexity to the term 'gene'. There are many different types of regulatory elements, and they generally operate in complex combinations. Some of them, such as enhancers, are nonspecific and influence any compatible promoter within their range.

Others, such as the promoters of polycistronic mRNAs, control the synthesis of several gene products. Furthermore, there is already an acceptable term ('operon') for a unit of gene expression (page 339 of ref. 3).

Should introns be considered as parts of genes? When first discovered, genes with introns were described as 'interrupted'. This implies that a gene consists only of its exons; otherwise it would be continuous. An 'exons only' definition highlights the evolutionary theory that new genes can be created by shuffling existing exons into new combinations. There is already an acceptable term ('transcription unit') for the entire region, including introns, between a promoter and a transcriptional terminator (page 366 of ref. 4). In cases of alternative splicing of primary transcripts, it has been suggested that, unless the protein products are very different, one gene can encode a series of protein isoforms (page 457 of ref. 4).

In my opinion, the single best molecular definition of the term 'gene' is the following: it is the nucleotide sequence that stores the information which specifies the order of the monomers in a final functional polypeptide or RNA molecule, or set of closely related isoforms. This definition is simple and concise. Geneticists can readily find other names for more complex genetic entities such as 'operons' and 'transcription units'.

Christopher D. Epp

College of Sciences,

Massey University,

Palmerston North,

New Zealand

1. Lodish, H. et al. J. Mol. Cell Biol., 3rd edn, 308-310 (Scientific

American Books, 1995)

2. Pennisi, E. \& Roush, W. Science 277, 34-37 (1997).

3. Lewin, B. Genes VI (Oxford Univ. Press, 1997).

4. Alberts, B. et al. Molecular Biology of the Cell, 3rd edn (Garland Publishing, 1994). vocabulary, yet it has no precise universally accepted molecular definition. 